\title{
Simian homologues of Epstein-Barr virus
}

\author{
Fred Wang*, Pierre Rivailler, Pasupuleti Rao and Young-gyu Gho
}

Department of Medicine, Brigham and Women's Hospital, Harvard Medical School, Boston, MA 02115, USA

\begin{abstract}
$\gamma$-Herpesviruses closely related to the Epstein-Barr virus (EBV) are known to naturally infect Old World non-human primates and are classified in the same lymphocryptovirus (LCV) genera. LCV infecting humans and Old World primates share similar biology, and recent studies have demonstrated that these viruses share a similar repertoire of viral genes. Surprisingly, the latent infection genes associated with cell growth transformation demonstrate the most striking sequence divergence, but the functional mechanisms for these genes are generally well conserved. The recent discovery of LCVs naturally infecting New World primates has rewritten the old paradigm of LCV host range restriction to humans and Old World non-human primates, so that these viruses are more widespread than previously believed. However, the New World LCV genome has significant and interesting differences from EBV and other Old World LGVs despite similar biological properties. Thus, the simian homologues of EBV can provide an important animal model for studying LGV pathogenesis, and the similarities and differences that have evolved among these related viruses can provide a unique perspective towards a better understanding of EBV.
\end{abstract}

Keywords: herpesvirus; primates; transformation; pathogenesis; lymphoma; evolution

\section{HISTORICAL PERSPECTIVE}

Infection of Old World non-human primates with Epstein-Barr virus (EBV) related lymphocryptoviruses (LCVs) was recognized in the early to mid-1970s. Investigators used the newly developed indirect immunofluorescence assay to detect antibodies cross-reactive to the EBV capsid antigen (VCA) in the serum of many Old World non-human primate species (Dunkel et al. 1972; Frank et al. 1976; Goldman et al. 1968; Kalter et al. 1972; Landon \& Malan 1971; Levy et al. 1971; Naito et al. 1971; Stevens et al. 1970; Werner et al. 1972). The failure to detect EBV crossreactive antibodies in New World primates suggested that LCVs were restricted to humans and non-human Old World primates. This pattern of evolution is very different from that of the Kaposi's sarcoma herpesvirus-related $\gamma$ herpesviruses in the rhadinovirus genera that were found later in humans, Old and New World non-human primates, and many non-primate species.

Subsequently, LCV-infected cell lines were established from healthy and diseased Old World non-human primates. One of the first simian LCVs identified was from baboons at the Institute of Experimental Pathology, USSR Academy of Medical Sciences at Sukhumi. An outbreak of lymphomas in this baboon colony was recognized, beginning in 1967 (Lapin 1974), and lymphoid cell lines containing herpes particles and expressing a crossreactive VGA were established from lymphomatous baboons (Djatchenko et al. 1976; Rabin et al. 1977). Simian LCV-infected lymphoblastoid cell lines were also established by other investigators using peripheral blood

\footnotetext{
*Author and address for correspondence: Channing Laboratory,

181 Longwood Avenue, Boston, MA 02115, USA

(fwang@rics.bwh.harvard.edu).
}

lymphocytes from normal, healthy baboons (Falk et al. 1976). LCV-infected cell lines have been established using lymphocytes from healthy and diseased chimpanzees (Gerber et al. 1976; Landon et al. 1968), gorillas (Neubauer et al. 1979), orang-utans (Rasheed et al. 1977), and various macaque species (Bocker et al. 1980; Fujimoto et al. 1990; Heberling et al. 1981; Lapin et al. 1985; Rangan et al. 1986). Simian LCV-infected cell lines produced virus that could immortalize B cells from autologous and closely related species in vitro (Falk et al. 1977; Fujimoto et al. 1990; Gerber et al. 1977; Ishida \& Yamamoto 1987; Neubauer et al. 1979; Rabin et al. 1977, 1978; Rangan et al. 1986). These cell lines also expressed a latent infection nuclear antigen that could be detected by immune simian sera, similar to the Epstein-Barr viral nuclear antigens (EBNA). But the simian latent infection nuclear antigens did not crossreact well with EBV immune human sera (Dillner et al. 1987; Gerber et al. 1976; Li et al. 1993; Ohno et al. 1977).

In vivo, LCV infection in non-human primates also resembles EBV infection in humans. Newborn animals are sero-positive for VCA antibodies due to maternal antibody transfer. They become sero-negative within four to six months after birth, and then most sero-convert again within the year, indicating a high prevalence of infection (Frank et al. 1976; Fujimoto \& Honjo 1991; Jenson et al. 2000; Landon \& Malan 1971; Rao et al. 2000). Lifelong antibody responses, the ability to recover simian LCVinfected B-cell lines from the peripheral blood of healthy animals, and the ability to detect virus shed from the oropharynx all indicate that a similar host-LCV relationship exists in human and non-human hosts (Moghaddam et al. 1998).

Shortly after the EBV genome was cloned, investigators addressed how similar the simian LCV genomes were to EBV. These studies revealed that the simian LCV 
genomes were organized in a collinear fashion with EBV (Heller \& Kieff 1981; Heller et al. 1981, 1982). Virtually all EBV DNA fragments were found to cross-react with viral DNA from baboon and chimpanzee LGVs with an overall homology of $40 \%$. It is interesting to note that two regions of the EBV genome did not cross-hybridize well to the simian LCV genomes, specifically the BamHI E and Nhet DNA fragments. At the time, it was not recognized that these regions encoded for the latent infection nuclear antigen 3 family and the latent infection membrane proteins 1 and 2, respectively. Subsequent studies have confirmed the more dramatic sequence divergence at these loci.

At the same time, several investigators tested whether non-human primates could serve as an experimental animal model for EBV infection. New World primates, e.g. cotton-top tamarins, inoculated with EBV developed either tumours or cleared the infection without establishing a persistent infection (Shope et al. 1973). However, there was little evidence for successful experimental infection of various Old World species such as macaques or baboons when inoculated with B95-8 or P3HR-1 EBV strains (Frank et al. 1976; Gerber et al. 1969; Levine et al. 1980). It is unclear if infection of Old World non-human primates failed due to cross-reactive immunity from natural LCV infection, the use of defective EBV isolates, or the route of inoculation. More recent studies suggest that there is a species restriction for LCV-induced B-cell immortalization that may also have contributed to the lack of successful experimental infection (Moghaddam et al. 1998). Interest in simian LCV gradually waned in the 1980s in favour of molecular techniques to study the EBV lytic and latent gene repertoire, regulation of viral gene expression, and mechanisms of viral gene induced transcription and signal transduction.

The need for an experimental animal model for EBV infection has been resurrected more recently as fundamental questions about EBV pathogenesis in vivo remain unanswered. Interest in simian $\mathrm{LCV}_{\mathrm{s}}$ has also been stimulated by the appearance of LCV-induced tumours arising in animals immunosuppressed by infection with simian immunodeficiency virus (SIV) (Feichtinger et al. 1990; Habis et al. 2000), similar to the development of EBV-positive B-cell lymphomas in AIDS patients.

Historically, this group of viruses has been given a variety of different names as they have been discovered, such as 'rhesus EBV' (rhesus macaque), 'cyno EBV' (cynomolgus macaque), Herpesvirus papio (baboon) and Herpesvirus pan (chimpanzee). Unfortunately, these names are often neither correct nor very specific. Epstein-Barr virus is the name applied to the LCV found in humansthe simian LCVs described subsequently, although similar to EBV, are genetically distinct. Many Old World non-human primates are infected with $\alpha$ - and $\beta$-herpesviruses as well as $\gamma$-herpesviruses, so that names such as Herpesvirus papio are not very specific. Official nomenclature recommended by the Study Group for the International Committee on the Taxonomy of Viruses (ICTV, see http://www.ncbi.nlm.nih.gov/ICTV/) uses a sequential number in order of discovery combined with the family or subfamily of the natural host, e.g. 'cercopithicine herpesvirus 15' for 'rhesus EBV' as the 15th herpesvirus found naturally to infect species in the family
Cercopithecidae. This system recognizes the unique identity of these various virus species, but fails to provide a very useful and easily recognizable name. Thus, we propose supporting the ICTV nomenclature while using the combination of the host species and LCV for vernacular usage, e.g. rhesus LGV, baboon LGV, chimpanzee LCV.

More recently, we have shown that rhesus macaques can be experimentally infected by oral inoculation with a rhesus LGV isolate (Moghaddam et al. 1997). Mimicking the natural route of inoculation results in acute and persistent LCV infection that resembles EBV infection of humans. Animals shed virus in oral secretions and harbour persistent, asymptomatic LCV infection in the peripheral blood, indicating a similar balance between immunological control and virus immune evasion to that observed in humans. These studies were made possible by (i) the isolation of a rhesus LCV isolate (Rangan et al. 1986), and (ii) development of specific pathogen-free macaque colonies that provide a routine source of LCVnaive animals (Desrosiers 1997). Thus, rhesus macaques and their naturally occurring LCV provide a model system for experimentally testing the role of specific viral genes for LCV pathogenesis in vivo.

A better understanding of the rhesus LCV is therefore essential for validating the relevance of rhesus LCV infection as a model for EBV infection in humans. In this chapter we will review what has been learned about the rhesus LCV genome, its similarity to EBV, the recent discovery of LCVs in New World primates, and the phylogenetic relationships of these various human and nonhuman LCVs.

\section{SIMIAN LYMPHOCRYPTOVIRUS LYTIC INFECTION GENES}

The sequencing of the rhesus LCV genome is nearly complete, and studies to date suggest that the EBV genes expressed during lytic infection are well conserved in the rhesus LCV. There appears to be a rhesus LCV homologue for each EBV lytic infection gene (P. Rivailler, Y. Cho and F. Wang, unpublished data). This is not surprising for genes such as DNA polymerase, other proteins important for DNA replication and structural proteins, given the conserved replication mechanisms among all herpesviruses. Other lytic genes unique to EBV among the herpesviruses, such as the major membrane glycoprotein (gp350) (Moghaddam et al. 1998) and Zebra transactivator (Jiang et al. 2000) are conserved in rhesus LCV. Functionally, the EBV Zebra transactivator can induce expression of rhesus LCV lytic infection gene expression, induce rhesus LCV DNA replication, and induce production of transforming rhesus LCV, reflecting the wellconserved nature of herpesvirus replication (Moghaddam et al. 1997). In addition, lytic genes with interesting but non-essential roles for EBV replication, such as BARF1 (CSF1R homologue) and BCRF1 (vIL-10) are also conserved in the rhesus LCV (Cho et al. 2001). These are especially important points for the validation of the rhesus LCV animal model since these genes are not essential for virus replication in vitro and are likely to be important for successful virus infection in vivo. The conservation of a $v I L$ 10 and CSF1R homologue suggests that these genes provide 
a similar biological advantage for rhesus LCV infection in macaques as for EBV infection in humans. It remains to be determined how these immunomodulatory genes are advantageous for LCV infection in vivo.

The rhesus LCV lytic infection genes common among herpesviruses are generally well conserved with 70-95\% similarity to the EBV homologues (Cho et al. 2001). Thus, it is not surprising that serum antibodies to lytic infection genes from humans and Old World primates frequently cross-react with each other. In humans, epitopes in the C-terminus of the small virus capsid antigen (BFRF3) are immunodominant (Van Grunsven et al. 1994). This region is moderately well conserved in the rhesus LCV small VCA, and an enzyme-linked immunoassay using a peptide from the C-terminus of the rhesus LCV BFRF3 homologue indicates that this is an immunodominant epitope in rhesus macaques and is an effective screening tool for identifying rhesus LCV infection (Rao et al. 2000).

The gp350 homologue is the least-well-conserved lytic infection gene identified to date, with only $49 \%$ homology (Moghaddam et al. 1998), perhaps because it is the 'newest' gene in the LGV lytic infection gene repertoire. The receptor binding site in gp350 is well conserved, suggesting that simian LCVs also use the same cellular receptor as EBV. It is known that both rhesus and baboon LCV can infect human B cells, consistent with the well-conserved nature of the membrane glycoproteins (Moghaddam et al. 1998). However, it is interesting to note that we have been unable to transform human B cells with either rhesus or baboon LCV despite a single published result to the contrary (Moghaddam et al. 1998). EBV can immortalize chimpanzee B cells and baboon LCV can immortalize rhesus macaque B cells, suggesting that growth transformation may be limited to more closely related Old World species. On the other hand, EBV can immortalize New World primate B cells.

The ability of simian LCV to infect, persist and replicate in, but not immortalize human B cells was demonstrated experimentally when baboon LCV was applied to human peripheral blood B cells from a donor with a high EBV viral load (Moghaddam et al. 1998). A large number of immortalized B cells were obtained, and all cell clones contained both baboon LCV and EBV. Thus, these cell lines were derived from the spontaneous outgrowth of EBV-infected B cells. Since, the multiplicity of infection of baboon LCV was sufficiently high to ensure infection of every EBV-infected cell, EBV-negative B cells were certainly infected with baboon LCV, but failed to become immortalized. The baboon LCV persisted, replicated and was fully transformation competent because virus could be rescued by infection and immortalization of rhesus macaque peripheral blood B cells. These experiments therefore demonstrate the well-conserved nature of the lytic infection genes, but indicate a species restriction for B-cell growth transformation, perhaps due to functional differences in the latent infection genes.

\section{SIMIAN LYMPHOCRYPTOVIRUS LATENT INFECTION GENES}

Simian LCV-infected B cells expressed nuclear antigens that could be detected by immune simian serum similar to the detection of EBNAs in EBV-infected B cells with immune human sera. The lack of significant crossreactivity and the lack of cross-hybridization with EBV DNA fragments encoding large blocks of latent infection genes suggested that there were more significant sequence differences among the simian latent infection genes as compared with the lytic infection genes. Cloning of the simian LCV latent infection gene homologues confirms that these genes are less well conserved than the lytic infection genes, but the simian LCVs appear to encode an identical repertoire of latent infection genes that function in a nearly identical manner as the EBV latent infection genes.

\section{(a) Latent membrane protein 1}

The rhesus and baboon LCV LMP-1 coding sequences were deduced from genomic DNA and a partial cDNA respectively (Franken et al. 1996). Both simian LMP-1 homologues also appear to be larger than the 386AA prototype B95-8 EBV LMP-1 sequence. The rhesus LCV LMP-1 contains 588 amino acids and the baboon LCV partial cDNA contains 389 amino acids and starts at a position homologous to residue 59 of EBV LMP-1. Both simian LMP-ls encode six hydrophobic domains well conserved with the transmembrane domains of LMP-1. However, the C-terminal cytoplasmic domains are strikingly divergent with no meaningful alignment overall. In addition, each LMP-1 sequence appears equidistant from each other; e.g. the rhesus LCV LMP-1 sequence is as distant from the baboon LCV LMP-1 as it is from the EBV LMP-1.

At the time, it was recognized that LMP-1 could induce nuclear factor $-\kappa \mathrm{B}(\mathrm{NF}-\kappa \mathrm{B})$ activity and expression of a broad number of B-cell activation genes (Hammarskjold \& Simurda 1992; Laherty et al. 1992; Wang et al. 1990) and that the LMP-1 C-terminal cytoplasmic domain was likely to be important for inducing NF- $\mathrm{BB}$ activity through interactions with tumour necrosis factor (TNF)-receptor associated factors (Mitchell \& Sugden 1995). The rhesus and baboon LCV LMP-ls could also induce NF- $\mathrm{\kappa B}$ activity and the adhesion molecule ICAM1 similar to the EBV LMP-1 (Franken et al. 1996). On closer inspection, there were two restricted regions of homology between the EBV and simian LMP-1s: (i) a sequence near the membrane proximal portion of the $\mathrm{C}$ terminal cytoplasmic domain; and (ii) the extreme distal end of the C-terminal cytoplasmic domain. The membrane proximal motif correlated with LMP-1 aminoacid residues 186-212, known to be part of the interaction domain with TNF receptor-associated factor 3 (TRAF3) in yeast two-hybrid assays (Mosialos et al. 1995). The 12 amino-acid core of this conserved proximal domain contained a PXQXT/S motif that was repeated with minor variations six times in the rhesus LCV LMP-1 and eight times in the baboon LCV LMP-1. Glutathione-Stransferase (GST) fusion proteins demonstrated that different 12 amino-acid repeat units were sufficient to interact with TRAF3 in GST pull-down assays. Four out of six motifs in the rhesus LCV LMP-1 and five out of eight motifs in the baboon LCV LMP-1 were capable of binding TRAF3. When these different TRAF3 binding sequences were aligned, it was evident that the PXQXT/ $\mathrm{S}$ motif was the only common element, and this was the 
first identification of a minimal PXQXT/S motif sufficient for TRAF interaction (Franken et al. 1996). It is interesting to note that the simian LCV LMP-1s are strikingly different from the EBV LMP-1 through the presence of multiple TRAF binding motifs in their C-terminal cytoplasmic domains. While the rhesus and baboon LCV LMP-1s induce similar levels of NF- $\mathrm{KB}$ activity, the potential significance of these multiple TRAF binding domains remains unclear and perhaps this difference in the composition of what is now referred to as TES-1 (transformation effector site 1) or CTAR-1 (cytoplasmic activating region 1) contributes to the species-specific restriction of LCV-induced B-cell immortalization. EBV LMP-1 function is known to require homotypic aggregation of the molecule in the cell membrane ('patching'), an effect which requires the multiple membrane spanning domains. With the rhesus and baboon LGV LMP-1s, their multiple TRAF binding motifs have the potential to bind more signalling molecules and thereby possibly to aggregate TRAF molecules in the absence of typical patching. Perhaps this is why the baboon LCV LMP-1 partial cDNA is still capable of inducing NF- $\mathrm{B}$ despite a truncation that could affect its membrane localization.

The homology in the distal C-terminal cytoplasmic domain was even more striking than the homology in TES-1. In the last 13 amino acids of each LMP-1 seven residues are conserved identically, five residues are similar, and a characteristic doublet of tyrosine residues is present in each. The distal C-terminus was recognized to

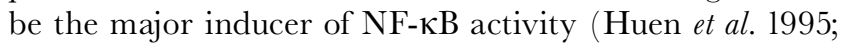
Mitchell \& Sugden 1995) and this well-conserved terminal sequence was subsequently shown to be essential for interaction with the TNF receptor-associated death domain (TRADD) adapter molecule (Izumi \& Kieff 1997). Similarly, this region (TES-2, CTAR-2) is important for EBV-induced B-cell immortalization (Kaye et al. 1995).

The rhesus LCV LMP-1 has also been shown to induce anchorage-independent growth and morphological changes in rodent fibroblasts similar to LMP-1 (Cho et al. 2001). The rhesus LCV LMP-1 is also able to induce activator protein 1 (AP-1) activity suggesting that it also recruits the jun N-terminal kinase (JNK) pathway similar to EBV LMP-1 (Cho et al. 2001). Thus, despite marked sequence heterogeneity there appears to be a strong selection for common signalling pathways in the EBV and Old World LCV LMP-ls.

\section{(b) Latent membrane protein 2}

Baboon and rhesus LCV homologues for the EBV LMP-2A were readily identified by conservation of a characteristic immunoreceptor tyrosine activated motif (ITAM) (Franken et al. 1995; Rivailler et al. 1999). The full-length baboon LCV LMP-2A was initially cloned from a cDNA library, and the rhesus LCV LMP-2A was deduced from the genomic sequence and confirmed by reverse transcriptase-polymerase chain reaction (RTPCR) analysis. Like LMP-1, the multiple (in this case 12) transmembrane domains are well conserved, and the cytoplasmic domain associated with signalling, i.e. the LMP-2A first exon encoding the N-terminal cytoplasmic domain, is significantly divergent among the different LCVs. Despite this sequence divergence, the ITAM and proline-rich domains that are important for interaction with protein tyrosine kinases (PTK) are conserved, and the simian LCV LMP-2s both induce PTKs and are themselves tyrosine phosphorylated.

Initially, it appeared that simian LCV might be different from EBV in the expression of a LMP-2B homologue since only a single LMP-2A transcript was detected on Northern blots (Franken et al. 1995) and since the rhesus LCV LMP-1 promoter region, where a putative LMP-2B first exon might be encoded, was very divergent from EBV (Rivailler et al. 1999). However, additional studies revealed that, like EBV, the rhesus LCV LMP-1 promoter could function in both directions and careful RT-PCR analysis demonstrated that a homologue for the EBV LMP-2B transcript is expressed in rhesus LGV-infected B cells (Rivailler et al. 1999). Like EBV, the rhesus LCV LMP-2B exon is non-coding, and since LMP-2B and LMP-2A are identical except for the first exons, LMP-2B appears to be a truncated form of LMP-2A.

It remains to be determined whether the rhesus $\mathrm{LCV}$ LMP-2A and LMP-2B genes are dispensable for B-cell growth transformation as they are for EBV. However, it is clear that there is a strong selective pressure for LMP-2A and LMP-2B that has been conserved among the human and Old World LCV. How LMP-2A might contribute to EBV pathogenesis in vivo is a question that can be addressed by genetic manipulation of rhesus LCV and infection of rhesus macaques with a rhesus LCV LMP-2A mutant virus. What and how LMP-2B might be contributing to LCV infection remains a mystery and may be more difficult to analyse given the difficulty in genetically altering the LMP-2B locus only, and in detecting LMP$2 \mathrm{~B}$ expression. However, the conservation of LMP-2B in simian LCV suggests that it will have an important role.

\section{(c) EBNA-1}

$E B N A-1$ is one of the most well conserved of the simian LCV latent infection genes (Blake et al. 1999; Yates et al. 1996). This was perhaps not unexpected given the EBNA1 protein's important role in episomal maintenance. Both baboon and rhesus LCVs encode smaller EBNA-1 homologues (476 amino acids and 511 amino acids, respectively, versus 641 amino acids for EBV EBNA-1), and this is due almost entirely to differences in the glycine-alanine repeat (GAR) domain. Where the EBV EBNA-1 contains 84 repeats of $\mathrm{a}_{1-3} \mathrm{~A}$ peptide over 252 amino acids, the rhesus LCV EBNA-1 contains four perfect repeats of a GAGGS motif preceded by three GAGGS repeats interspersed with 12 additional amino acids forming seven glycine-alanine-rich repeats within a 47 amino-acid stretch. The baboon LCV EBNA-1 contains seven perfect repeats of a similar GAGAGGS motif. In all three species, the GAR domain is flanked on both sides by glycine-arginine (GR)-rich regions. The remainder of the EBNA-1 C-terminus containing the DNA-binding and dimerization domain is relatively well conserved with $63 \%$ and 53\% amino-acid identity between EBV and rhesus or baboon LCV, respectively. The baboon and rhesus LCV EBNA-1 homologues can both support EBV ori-P-dependent plasmid replication and maintenance, as might be expected from the strong conservation of the GR-rich regions important for DNA linking and of the C-terminus important for DNA binding. 
The smaller and simpler simian LCV GAR domains were interesting because the EBV EBNA-1 GAR had been reported to be important for inhibiting antigen presentation in cis (Levitskaya et al. 1995). Thus, similar experiments were performed to identify whether this immunomodulatory function had been conserved in the simian LCV EBNA-1 homologues (Blake et al. 1999). First, the baboon and rhesus LCV GARs were used to replace the GAR in EBV EBNA-1. The chimeric proteins containing the unique regions EBV EBNA-1 and the simian LGV GAR efficiently processed and presented an EBV EBNA-1 cytotoxic T lymphocyte (CTL) epitope located downstream of the GAR, when expressed in human cells by vaccinia infection and challenged with a human EBNA-1 specific CTL clone. To test whether a block to antigen presentation might only occur in simian cells, simian B-cell lines were co-infected with vaccinia vectors expressing the chimeric EBNA-1 molecules and the restricting HLA molecule necessary for presentation of the EBNA-1 specific CTL epitope. However, these co-infected simian targets were again efficiently killed by the EBNA-1 specific CTL. Finally, to test whether a block to antigen presentation might require a simian background in both effector and target cells, a rhesus CTL clone specific for a SIV epitope was used in conjunction with the autologous rhesus B-cell line as the target cell. In this case, the SIV CTL epitope sequence was inserted into the wild-type rhesus LCV EBNA-1, both upstream and downstream of the GAR to address any potential positional effect. However, in all cases the SIV epitope was efficiently presented, indicating that the simian LCV EBNA-1 homologue has no inhibitory effect on antigen presentation.

These observations call into question whether immune evasion per se is the primary function of the GAR. The EBV EBNA-1 GAR clearly can inhibit antigen presentation and the immune evasion capability of EBV EBNA-1 may be a relatively recent acquisition in evolutionary terms, perhaps as a by-product of a more fundamental and more widely conserved property of the LCV GAR domain. EBV persistence in resting lymphocytes in the memory B-cell population may be sufficient to afford these cells immunological protection, since resting cells do not express the co-stimulatory molecules upon which immune T-cell activation depends and are likely to have much reduced antigen presenting capacity compared with activated proliferating B lymphoblasts. This may also help to explain why other potentially immunogenic viral antigens such as LMP-2A, thought to be expressed in the EBV-positive reservoir of resting $\mathrm{B}$ cells, could be sustained without alerting the attentions of the CTL response. Conservation of a type I latency promoter in simian LCV similar to the EBV BamHI Q promoter (Ruf et al. 1999) suggests that downregulation of latent gene expression may be a more fundamental mechanism for immune evasion common among all LCVs.

\section{(d) $E B N A-2$}

The $E B N A-2$ homologues are one of the least wellconserved genes among simian LCV $(26.3 \%$ and $26.5 \%$ similarity for baboon and rhesus LCV, respectively, versus EBV EBNA-2) (Cho et al. 1999; Ling \& Hayward 1995; Peng et al. 2000). Again, one of the surprising findings is that these genes are equidistant from each other, i.e. the baboon and rhesus LCV $E B \mathcal{N} A-2 \mathrm{~s}$ are not any more similar to each other $(26.7 \%$ similarity $)$ than they are to EBV $E B N A-2$. This recurring theme among the latent infection genes suggests that these B-cell transformationassociated genes have evolved more recently from a relatively diverse gene pool and only certain sequence motifs have evolved due to the selection for common biological processes important for B-cell immortalization. Consistent with this model, interaction with the transcription factor RBP-J $\kappa$ and amino-acid residues important for this interaction have been conserved in the simian LCV EBNA-2 homologues despite their striking overall sequence divergence (Ling \& Hayward 1995).

An equally curious quirk of nature is the observation that there appear to be two different types of rhesus LCV defined by polymorphisms in the $E B \mathcal{N} A-2$ gene as there are in EBV (Cho et al. 1999). This was discovered serendipitously when a rhesus LCV-positive cell line was isolated from a lymphomatous, SIV-infected rhesus macaque at the New England Regional Primate Research Center (NERPRG). The EBNA-2 monoclonal antibody, PE2, detected an epitope in the acidic transactivating domain that is well conserved in the simian LCV EBNA-2 homologues, and an EBNA-2 immunoblot with PE2 detected an EBNA-2 of a different size from the prototype LCL8664 rhesus LCV isolate found originally at the Tulane Regional Primate Research Center. When the two rhesus LCV EBNA-2 sequences were compared they were significantly different from each other $(41.1 \%$ amino-acid similarity), suggesting a relationship similar to that between the type 1 and type 2 EBV EBNA-2s (54.7\% amino-acid similarity). Even more striking was the similar domain structure between the EBNA-2 types. Type 1 and type 2 EBV EBNA-2s have most similarity in the N-terminal negatively charged domain, RBP-J $\kappa$ binding domain, and acidic transactivating domain, and are most dissimilar in a divergent domain defined in the middle of the protein. The two types of rhesus LCV EBNA-2 have a similar relationship, with more conserved functional domains and a divergent domain in the middle of the protein. A weaker B-cell transformation phenotype has been associated with type $2 \mathrm{EBV}$ and the type 2 EBNA-2 specifically, and the NERPRG rhesus LCV is noted to immortalize rhesus B cells more poorly. However, it is unclear how this phenotype may confer a selective advantage for LCV infection. One presumes that this polymorphism must confer some advantage for LCV infection in order to be conserved during LGV evolution in two different species.

\section{(e) EBNA-LP}

An EBNA leader protein (EBNA-LP) homologue is expressed in baboon and rhesus LCV-infected B cells and both include exons derived from the major internal repeat sequences and downstream unique sequences (Peng et al. 2000). The simian LCV homologues are well conserved with $61 \%$ and $64 \%$ homology, respectively, in the exons derived from the internal repeat sequence and $51 \%$ identity in the unique exons. The baboon and rhesus LCV EBNALP homologues are also functionally conserved in their ability to co-activate transcription with either the EBV or simian LCV EBNA-2s. The sequence comparisons also highlight four out of eight potential serine phosphorylation 


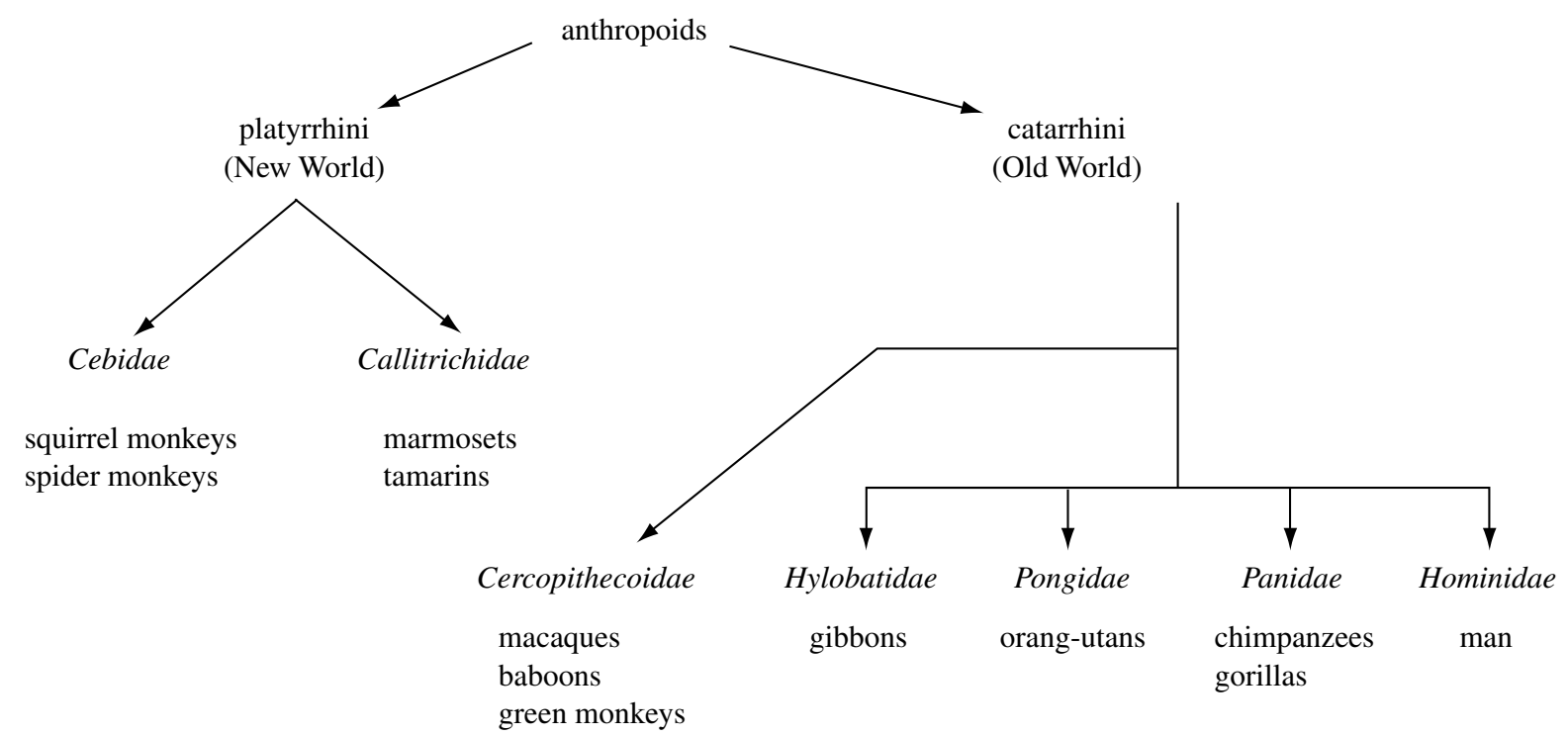

Figure 1. Primate taxonomy. Major families of New World and Old World primates are shown in italics with representative species listed below.

sites that may be functionally important and that are conserved among all three species.

\section{(f) EBNA-3A, $-3 B$ and $-3 C$}

The weak homology of the rhesus LCV EBNA-3 homologues to EBNA-3A, -3B and -3C (29.4, 29.1 and $31.0 \%$ amino-acid similarity, respectively; Jiang et al. 2000) was predicted by the failure of the EBV BamHl E DNA fragment to cross-hybridize with other LCV genomes. Although the function of these EBV latent infection genes is poorly understood, their interactions with the transcription factor RBP-J $\kappa$ and EBNA-3C's ability to act as a transcriptional transactivator have been conserved in the rhesus LCV homologues. Also conserved are repeat structures in each of these genes, although in most cases the repeat sequences themselves are not conserved between rhesus LCV and EBV. The importance of these repeat structures remains a mystery.

\section{NEW WORLD LYMPHOCRYPTOVIRUSES}

The classic paradigm has been that the LCV host range is restricted to humans and Old World primates since serological evidence for LCV infection (i.e. antibodies crossreactive against Old World $\mathrm{LGVs}$ ) could not be found in New World primates (Frank et al. 1976). However, this paradigm was recently challenged by investigators at the Wisconsin Regional Primate Research Center (WRPRG). Ramer and colleagues noted an unusually high incidence of spontaneous B-cell lymphomas in common marmosets (Callithrix jacchus) (Ramer et al. 2000). Because of the association between EBV and B-cell lymphomas in humans, these investigators looked for evidence of a herpesvirus infection by PCR using degenerative primers capable of amplifying diverse herpesvirus DNA polymerase and terminase sequences. They were able to amplify novel sequences that were most closely related to EBV from both diseased and healthy common marmosets. However, the lack of a virus isolate in vitro limited further characterization of this putative LCV.

We have subsequently isolated a herpesvirus from common marmoset lymphomas (Cho et al. 2001). Cloning and sequencing nearly two-thirds of the viral genome has definitively identified that the WRPRC common marmosets are infected with a $\gamma$-herpesvirus belonging to the LCV genera (Cho et al. 2001). In accordance with ICTV nomenclature, this virus is identified as CalHV-3. Additional studies demonstrate that CalHV-3 infection is prevalent in the peripheral blood lymphocytes from a majority of healthy common marmosets at the NERPRC, indicating that CalHV-3 infection is indigenous to common marmosets and not an accidental infection from Old World primates at the WRPRC. An LCV-like virus related to CalHV-3 can also be detected in the peripheral blood lymphocytes from squirrel monkeys (Saimiri sciureus), which belong to the other major family of New World primates (figure 1), indicating that LCV infection is likely to be common to most, if not all, New World primate species.

While the CalHV-3 sequence is not yet complete, it has already revealed some striking differences from EBV and other Old World LCVs.

(i) Three unique genes with no homology to other viral or cell genes are encoded by CalHV-3 and have been named $C 1, C 2$ and $C 3$.

(a) C1 is located near the terminal repeats at a position similar to that of $K 1$ and $L M P-1$, the viral oncogenes in Kaposi's sarcoma-associated herpesvirus (KSHV) and EBV. Cl also has the ability to transform growth of rodent fibroblasts, and has a secondary structure similar to that of LMP-1, with a short N-terminal cytoplasmic domain, six hydrophobic transmembrane domains and a long Cterminal cytoplasmic domain. In contrast to the EBV LMP-1 and homologues found in Old World LCV, the motifs that bind TNF-receptor associated adapter molecules in the LMP-1 effector site 1 TES-1 
(PXQXS/T motif) and TES-2 (PXQXXYYD/A) are not conserved. However, C1 can still induce NF$\kappa \mathrm{B}$ activity to similar levels as the LMP-ls and appears to interact with the TNF receptor-associated factors (TRAFs) through different TRAF consensus binding motifs repeated multiple times in the $\mathrm{Cl}$ Cterminus. Thus, there has been biological selection for a common molecular pathway of signal transduction. Interestingly, the CalHV-3 Cl differs from the EBV and Old World LCV LMP-1s by its inability to activate AP-1 activity.

(b) C2 is a novel CalHV-3 gene that is encoded in place of a putative membrane glycoprotein, BILF2. C2 appears to be a transmembrane protein with immunoglobulin domains but, like BILF2, there are few clues as to the functional role for these gene products.

(c) C3 is a novel CalHV-3 gene that is located between CalHV-3 open reading frames encoding the gp350 and Zebra homologues. Thus, this single CalHV-3 gene is a positional homologue for the $E B \mathcal{N} A-3 A,-3 B$ and $-3 C$ genes encoded in EBV and other Old World LCVs. C3 has no homology to the EBNA-3s or other viral or cell genes. One hypothesis is that $C 3$ is a functional homologue for the three EBNA-3s, and that the three EBNA-3s evolved as gene duplication events in EBV and Old World LCV. Since CalHV-3 efficiently immortalizes marmoset lymphocytes and C3 is a positional homologue for EBV genes essential for growth transformation, C3 is likely to be an important viral gene.

(ii) CalHV-3 encodes an EBNA-1 homologue that is well conserved in the C-terminal DNA binding domain and GR-rich domains important for DNA linking. This well-conserved mechanism for episomal maintenance reflects the close relationship between CalHV-3 and the LCV genera. It is also interesting to note that there is essentially no GAR region in the CalHV-3 EBNA-1 homologue, but persistent infection and immune evasion is a common feature of infection with CalHV-3 and other LCVs. Thus, the inhibitory effects on antigen presentation by the EBNA-1 GAR appears to be a more recent evolutionary event limited to the human LCV.

(iii) CalHV-3 also lacks some genes that are conserved in EBV and other Old World LCV, e.g. BARF1 and $L M P$-2. BARF1 is a secreted homologue of the colony stimulating factor 1 receptor that is highly conserved in EBV and rhesus LGV (Cho et al. 2001; Strockbine et al. 1998). BARF1 is expressed during lytic viral replication, but is not essential for virus replication in tissue culture (Cohen \& Lekstrom 1999). Thus, this immunomodulatory viral gene product is most likely to be of importance for immune evasion and persistent infection in vivo, but appears to be unnecessary for CalHV-3 infection. No LMP-2 homologue is apparent by either basic local alignment search tool search or specific searches for ITAM sequences in the CalHV-3 sequence. It is unclear whether LMP-2 function is dispensable for marmoset LCV infection, or if CalHV-3 has developed an alternative strategy for achieving function.
These differences indicate that the New World LCVs are more distant from EBV and the Old World LCVs, but how these groups of related viruses evolved remains to be explained. However, LCV infection in humans, Old World and New World primates are unified by their common biological properties: (i) B-cell immortalization in vitro; (ii) persistent, asymptomatic infection in peripheral blood lymphocytes; (iii) high prevalence of infection in adult populations; and (iv) association with malignant transformation, e.g. B-cell lymphomas. Even the different pathogenetic mechanisms of lymphomagenesis are conserved in human and non-human primates; for example, the development of LCV-positive B-cell lymphomas in AIDS patients and in SIV-infected macaques, and the spontaneous development of LCV-positive B-cell lymphomas in relatively healthy hosts as in Burkitt's lymphoma patients and in CalHV-3-infected common marmosets.

It is unclear why the New World LCVs have a different repertoire of viral genes to mediate these similar biological events, but the differences provide a unique perspective. Could the CalHV-3 C3 be functionally equivalent to the three EBV EBNA-3s? Will the functional properties of $C 3$ be more evident as a single gene? What caused the apparent gene duplication resulting in three EBNA-3s? If $B A R F 1$ is an immunomodulatory gene, why is it not required for persistent CalHV-3 infection?

\section{CONCLUSION}

Our understanding of the EBV relatives in the LCV genera has increased significantly with the discovery and characterization of these Old and New World viruses. LGVs are clearly a larger group of viruses than previously believed and are a group of viruses that has undergone a still poorly understood process of evolution. However, the power of biological selection is evident among these different viruses, and these simian LCVs provide a unique opportunity for investigation. The relatively strong conservation between EBV and rhesus LCV makes the rhesus macaque an ideal animal model for analysing the pathogenesis of LCV infection in vivo with genetically altered viruses. On the other hand, the more divergent genome of the New World LCV provides an opportunity both to examine how nature has developed a slightly different approach to a similar quest for a persistent host-pathogen relationship and to identify mechanisms fundamentally important for LCV pathogenesis.

\section{REFERENCES}

Blake, N. W. (and 10 others) 1999 Inhibition of antigen presentation by the glycine/alanine repeat domain is not conserved in simian homologues of Epstein-Barr virus nuclear antigen 1. 7. Virol. 73, 7381-7389.

Bocker, J. F., Tiedemann, K. H., Bornkamm, G. W. \& zur Hausen, H. 1980 Characterization of an EBV-like virus from African green monkey lymphoblasts. Virology 101, 291-295.

Cho, Y. G., Gordadze, A. V., Ling, P. D. \& Wang, F. 1999 Evolution of two types of rhesus lymphocryptovirus similar to type 1 and type 2 Epstein-Barr virus. 7. Virol. 73, 9206-9212.

Cho, Y., Ramer, J., Rivailler, P., Quink, C., Garber, R. L., Beier, D. \& Wang, F. 2001 Epstein-Barr related herpesvirus from marmoset lymphomas. Proc. Natl Acad. Sci. USA 98, 1224-1229. 
Cohen, J. I. \& Lekstrom, K. 1999 Epstein-Barr virus BARFl protein is dispensable for B-cell transformation and inhibits alpha interferon secretion from mononuclear cells. F. Virol. 73, $7627-7632$.

Desrosiers, R. C. 1997 The value of specific pathogen-free rhesus monkey breeding colonies for AIDS research. AIDS Res. Hum. Retroviruses 13, 5-6.

Dillner, J., Rabin, H., Letvin, N., Henle, W., Henle, G. \& Klein, G. 1987 Nuclear DNA-binding proteins determined by the Epstein-Barr virus-related simian lymphotropic herpesviruses $H$. gorilla, H. pan, H. pongo and H. papio. F. Gen. Virol. 68, 1587-1596.

Djatchenko, A. G., Kakubava, V. V., Lapin, B. A., Agrba, V. Z., Yakovleva, L. A. \& Samilchuk, E. I. 1976 Continuous lymphoblastoid suspension cultures from cells of haematopoietic organs of baboons with malignant lymphoma-biological characterization and biological properties of the herpes virus associated with culture cells. Exp. Pathol. 12, 163-168.

Dunkel, V. C., Pry, T. W., Henle, G. \& Henle, W. 1972 Immunofluorescence tests for antibodies to Epstein-Barr virus with sera of lower primates. F. Natl Cancer Inst. 49, 435-440.

Falk, L., Deinhardt, F., Nonoyama, M., Wolfe, L. G. \& Bergholz, C. 1976 Properties of a baboon lymphotropic herpesvirus related to Epstein-Barr virus. Int. F. Cancer 18, 798-807.

Falk, L. A., Henle, G. Henle, W. Deinhardt, F. \& Schudel, A. 1977 Transformation of lymphocytes by Herpesvirus papio. Intl 7. Cancer 20, 219-226.

Feichtinger, H., Putkonen, P., Parravicini, C., Li, S. T., Kaya, E. E., Bottiger, D. \& Biberfeld, P. 1990 Malignant lymphomas in cynomolgus monkeys infected with simian immunodeficiency virus. Am. F. Pathol. 137, 1311-1315.

Frank, A., Andiman, W. A. \& Miller, G. 1976 Epstein-Barr virus and nonhuman primates: natural and experimental infection. Adv. Cancer Res. 23, 171-201.

Franken, M., Annis, B., Ali, A. N. \& Wang, F. 1995 5' coding and regulatory region sequence divergence with conserved function of the Epstein-Barr virus LMP2A homolog in Herpesvirus papio. F. Virol. 69, 8011-8019.

Franken, M., Devergne, O., Rosenzweig, M., Annis, B., Kieff, E. \& Wang, F. 1996 Comparative analysis identifies conserved tumor necrosis factor receptor-associated factor 3 binding sites in the human and simian Epstein-Barr virus oncogene LMP1. F. Virol. 70, 7819-7826.

Fujimoto, K. \& Honjo, S. 1991 Presence of antibody to CynoEBV in domestically bred cynomolgus monkeys (Macaca fascicularis). 7. Med. Primatol. 20, 42-45.

Fujimoto, K., Terato, K., Miyamoto, J., Ishiko, H., Fujisaki, M., Cho, F. \& Honjo, S. 1990 Establishment of a B-lymphoblastoid cell line infected with Epstein-Barr-related virus from a cynomolgus monkey (Macaca fascicularis). 7. Med. Primatol. 19, 21-30.

Gerber, P., Branch, J. W. \& Rosenblum, E. N. 1969 Attempts to transmit infectious mononucleosis to rhesus monkeys and marmosets and to isolate herpes-like virus. Proc. Soc. Exp. Biol. Med. 130, 14-19.

Gerber, P., Pritchett, R. F. \& Kieff, E. D. 1976 Antigens and DNA of a chimpanzee agent related to Epstein-Barr virus. $\mathcal{F}$. Virol. 19, 1090-1099.

Gerber, P., Kalter, S. S., Schidlovsky, G., Peterson, W. J. \& Daniel, M. D. 1977 Biologic and antigenic characteristics of Epstein-Barr virus-related herpesviruses of chimpanzees and baboons. Int. 7. Cancer 20, 448-459.

Goldman, M., Landon, J. C. \& Reisher, J. I. 1968 Fluorescent antibody and gel diffusion reactions of human and chimpanzee sera with cells cultured from Burkitt tumors and normal chimpanzee blood. Cancer Res. 28, 2489-2495.
Habis, A., Baskin, G., Simpson, L., Fortgang, I., MurpheyCorb, M. \& Levy, L. S. 2000 Rhesus lymphocryptovirus infection during the progression of SAIDS and SAIDSassociated lymphoma in the rhesus macaque. AIDS Res. Hum. Retroviruses 16, 163-171.

Hammarskjold, M. L. \& Simurda, M. C. 1992 Epstein-Barr virus latent membrane protein transactivates the human immunodeficiency virus type 1 long terminal repeat through induction of NF-kappa B activity. F. Virol. 66, 6496-6501.

Heberling, R. L., Bieber, C. P. \& Kalter, S. S. 1981 Establishment of a lymphoblastoid cell line from a lymphomous cynomolgus monkey. In Advances in comparative leukemia research. (ed. D. S. Yohn \& J. R. Blakeslee), pp. 385-386. Amsterdam: Elsevier.

Heller, M. \& Kieff, E. 1981 Collinearity between the DNAs of Epstein-Barr virus and Herpesvirus papio. F. Virol. 37, 821-826.

Heller, M., Gerber, P. \& Kieff, E. 1981 Herpesvirus papio DNA is similar in organization to Epstein-Barr virus DNA. F. Virol. 37, 698-709.

Heller, M., Gerber, P. \& Kieff, E. 1982 DNA of Herpesvirus pan, a third member of the Epstein-Barr virus-Herpesvirus papio group. F. Virol. 41, 931-939.

Huen, D. S., Henderson, S. A., Groom-Carter, D. \& Rowe, M. 1995 The Epstein-Barr virus latent membrane protein-1 (LMPl) mediates activation of NF-kappa B and cell surface phenotype via two effector regions in its carboxy-terminal cytoplasmic domain. Oncogene 10, 549-560.

Ishida, T. \& Yamamoto, K. 1987 Survey of nonhuman primates for antibodies reactive with Epstein-Barr virus (EBV) antigens and susceptibility of their lymphocytes for immortalization with EBV. 7. Med. Primatol. 16, 359-371.

Izumi, K. M. \& Kieff, E. D. 1997 The Epstein-Barr virus oncogene product latent membrane protein 1 engages the tumor necrosis factor receptor-associated death domain protein to mediate B lymphocyte growth transformation and activate NF-kappaB. Proc. Natl Acad. Sci. USA 94, 12 592-12 597.

Jenson, H. B., Ench, Y., Gao, S. J., Rice, K., Carey, D., Kennedy, R. C., Arrand, J. R. \& Mackett, M. 2000 Epidemiology of Herpesvirus papio infection in a large captive baboon colony: similarities to Epstein-Barr virus infection in humans. F. Infect. Dis. 181, 1462-1466.

Jiang, H., Cho, Y. G. \& Wang, F. 2000 Structural, functional, and genetic comparisons of Epstein-Barr virus nuclear antigen $3 \mathrm{~A}, 3 \mathrm{~B}$, and $3 \mathrm{C}$ homologues encoded by the rhesus lymphocryptovirus. F. Virol. 74, 5921-5932.

Kalter, S. S., Heberling, R. L. \& Ratner, J. J. 1972 EBV antibody in sera of non-human primates. Nature 238, 353-354.

Kaye, K. M., Izumi, K. M., Mosialos, G. \& Kieff, E. 1995 The Epstein-Barr virus LMPl cytoplasmic carboxy terminus is essential for B-lymphocyte transformation; fibroblast cocultivation complements a critical function within the terminal 155 residues. F. Virol. 69, 675-683.

Laherty, G. D., Hu, H. M., Opipari, A. W., Wang, F. \& Dixit, V. M. 1992 The Epstein-Barr virus LMPl gene product induces A20 zinc finger protein expression by activating nuclear factor kappa B. F. Biol. Chem. 267, 24 157-24 160.

Landon, J. C. \& Malan, L. B. 1971 Seroepidemiologic studies of Epstein-Barr virus antibody in monkeys. 7. Natl Cancer Inst. 46, 881-884.

Landon, J. C., Ellis, L. B., Zeve, V. H. \& Fabrizio, D. P. 1968 Herpes-type virus in cultured leukocytes from chimpanzees. 7. Natl Cancer Inst. 40, 181-192.

Lapin, B. A. 1974 The epidemiologic and genetic aspect of an outbreak of leukemia among baboons of the Sukhumi monkey colony in Dutcher and Chieco-Bianchi. Unifying concepts of leukemia. Biblphyl. Hematol. 39, 263-268.

Lapin, B. A., Timanovskaya, V. V. \& Yakovleva, L. A. 1985 Herpesvirus HVMA: a new representative in the group of the 
EBV-like B-lymphotropic herpesviruses of primates. Hamatol. Bluttransfus. 29, 312-313.

Levine, P. H., Leiseca, S. A., Hewetson, J. F., Traul, K. A., Andrese, A. P., Granlund, D. J., Fabrizio, P. \& Stevens, D. A. 1980 Infection of rhesus monkeys and chimpanzees with Epstein-Barr virus. Arch. Virol. 66, 341-351.

Levitskaya, J., Coram, M., Levitsky, V., Imreh, S., SteigerwaldMullen, P. M., Klein, G., Kurilla, M. G. \& Masucci, M. G. 1995 Inhibition of antigen processing by the internal repeat region of the Epstein-Barr virus nuclear antigen-1. Nature 375, 685-688.

Levy, J. A., Levy, S. B., Hirshaut, Y., Kafuko, G. \& Prince, A. 1971 Presence of EBV antibodies in sera from wild chimpanzees. Nature 233, 559-560.

Li, S. L., Feichtinger, H., Kaaya, E., Migliorini, P., Putkonen, P., Biberfeld, G., Middeldorp, J. M., Biberfeld, P. \& Ernberg, I. 1993 Expression of Epstein-Barr-virus-related nuclear antigens and B-cell markers in lymphomas of SIV-immunosuppressed monkeys. Int. F. Cancer 55, 609-615.

Ling, P. D. \& Hayward, S. D. 1995 Contribution of conserved amino acids in mediating the interaction between EBNA2 and CBF1/RBPJк. F. Virol. 69, 1944-1950.

Mitchell, T. \& Sugden, B. 1995 Stimulation of NF-kappa Bmediated transcription by mutant derivatives of the latent membrane protein of Epstein-Barr virus. 7. Virol. 69, 2968-2976.

Moghaddam, A., Rosenzweig, M., Lee-Parritz, D., Annis, B., Johnson, R. P. \& Wang, F. 1997 An animal model for acute and persistent Epstein-Barr virus infection. Science 276, 2030-2033.

Moghaddam, A., Koch, J., Annis, B. \& Wang, F. 1998 Infection of human B lymphocytes with lymphocryptoviruses related to Epstein-Barr virus. F. Virol. 72, 3205-3212.

Mosialos, G., Birkenbach, M., Yalamanchili, R., VanArsdale, T., Ware, C. \& Kieff, E. 1995 The Epstein-Barr virus transforming protein LMPl engages signaling proteins for the tumor necrosis factor receptor family. Cell 80, 389-399.

Naito, M., Ono, K., Doi, T., Kato, S. \& Tanabe, S. 1971 Antibodies in human and monkey sera to herpes-type virus from a chicken with Marek's disease and to EB virus detected by the immunofluorescence test. Biken f. 14, 161-166.

Neubauer, R. H., Rabin, H., Strnad, B. C., Nonoyama, M. \& Nelson, R. W. 1979 Establishment of a lymphoblastoid cell line and isolation of an Epstein-Barr-related virus of gorilla origin. 7. Virol. 31, 845-848.

Ohno, S., Luka, J., Falk, L. \& Klein, G. 1977 Detection of a nuclear, EBNA-type antigen in apparently EBNA-negative Herpesvirus papio (HVP)-transformed lymphoid lines by the acid-fixed nuclear binding technique. Int. F. Cancer 20, 941-946.

Peng, R., Gordadze, A. V., Fuentes Panana, E. M., Wang, F., Zong, J., Hayward, G. S., Tan, J. \& Ling, P. D. 2000 Sequence and functional analysis of EBNA-LP and EBNA2 proteins from nonhuman primate lymphocryptoviruses. $\mathcal{F}$. Virol. 74, 379-389.

Rabin, H., Neubauer, R. H., Hopkins, R. F., Dzhikidze, E. K., Shevtsova, Z. V. \& Lapin, B. A. 1977 Transforming activity and antigenicity of an Epstein-Barr-like virus from lympho- blastoid cell lines of baboons with lymphoid disease. Intervirology 8, 240-249.

Rabin, H., Neubauer, R. H., Hopkins, R. D. \& Rasheed, S. 1978 In vitro lymphocyte transformation by Epstein-Barr virus (EBV)-like viruses isolated from Old-World non-human primates. IARC Sci. Publ. 24, 553-557.

Ramer, J. G., Garber, R. L., Steele, K. E., Boyson, J. F., O'Rourke, G. \& Thomson, J. 2000 Fatal lymphoproliferative disease associated with a novel gammaherpesvirus in captive population of common marmosets. Comp. Med. 50, 59-68.

Rangan, S. R., Martin, L. N., Bozelka, B. E., Wang, N. \& Gormus, B. J. 1986 Epstein-Barr virus-related herpesvirus from a rhesus monkey (Macaca mulatta) with malignant lymphoma. Int. F. Cancer 38, 425-432.

Rao, P., Jiang, H. \& Wang, F. 2000 Cloning of the rhesus lymphocryptovirus viral capsid antigen and EBERs homologues and use in diagnosis of acute and persistent infection. $\mathcal{F}$. Clin. Microbiol. 38, 3219-3225.

Rasheed, S., Rongey, R. W., Bruszweski, J., Nelson-Rees, W. A., Rabin, H., Neubauer, R. H., Esra, G. \& Gardner, M. B. 1977 Establishment of a cell line with associated Epstein-Barr-like virus from a leukemic orangutan. Science 198, 407-409.

Rivailler, P., Quink, C. \& Wang, F. 1999 Strong selective pressure for evolution of an Epstein-Barr virus LMP2B homologue in the rhesus lymphocryptovirus. F. Virol. 73, 8867-8872.

Ruf, I. K., Moghaddam, A., Wang, F. \& Sample, J. 1999 Mechanisms that regulate Epstein-Barr virus EBNA-1 gene transcription during restricted latency are conserved among lymphocryptoviruses of Old World primates. F. Virol. 73, 1980-1989.

Shope, T., Dechairo, D. \& Miller, G. 1973 Malignant lymphoma in cotton-top marmosets following inoculation of EpsteinBarr virus. Proc. Natl Acad. Sci. USA 70, 2487-2491.

Stevens, D. A., Pry, T. W., Blackham, E. A. \& Manaker, R. A. 1970 Comparison of antigens from human and chimpanzee herpes-type virus-infected hemic cell lines. Proc. Soc. Exp. Biol. Med. 133, 678-683.

Strockbine, L. D., Cohen, J. I., Farrah, T., Lyman, S. D., Wagener, F., DuBose, R. F., Armitage, R. J. \& Spriggs, M. K. 1998 The Epstein-Barr virus BARFl gene encodes a novel, soluble colony-stimulating factor-1 receptor. F. Virol. 72, 4015-4021.

Van Grunsven, W. M., Spaan, W. J. \& Middeldorp, J. M. 1994 Localization and diagnostic application of immunodominant domains of the BFRF3-encoded Epstein-Barr virus capsid protein. F. Infect. Dis. 170, 13-19.

Wang, F., Gregory, C., Sample, C., Rowe, M., Liebowitz, D., Murray, R., Rickinson, A. \& Kieff, E. 1990 Epstein-Barr virus latent membrane protein $(\mathrm{LMPl})$ and nuclear proteins 2 and $3 \mathrm{C}$ are effectors of phenotypic changes in $\mathrm{B}$ lymphocytes: EBNA-2 and LMPl cooperatively induce CD23. F. Virol. 64, 2309-2318.

Werner, J., Henle, G., Pinto, C. A., Haff, R. F. \& Henle, W. 1972 Establishment of continuous lymphoblast cultures from leukocytes of gibbons (Hylobates lar). Int. F. Cancer 10, 557-567.

Yates, J. L., Camiolo, S. M., Ali, S. \& Ying, A. 1996 Comparison of the EBNAl proteins of Epstein-Barr virus and Herpesvirus papio in sequence and function. Virology 222, 1-13. 
